\title{
Electromagnetic shielding effectiveness of carbon fibre reinforced composites
}

\author{
D. Munallia,*, G. Dimitrakis ${ }^{\mathrm{b}}$, D. Chronopoulos ${ }^{\mathrm{a}}$, S. Greedy ${ }^{\mathrm{c}}$, A. Long ${ }^{\mathrm{d}}$ \\ ${ }^{a}$ Institute for Aerospace Technology $\& 5$ The Composites Research Group, The University of Nottingham, Nottingham, \\ $N G 7$ 2RD, UK \\ ${ }^{b}$ George Green Institute for Electromagnetics Research, Department of Chemical and Environmental Engineering, The \\ University of Nottingham, NG7 2RD, UK \\ ${ }^{c}$ George Green Institute for Electromagnetics Research, School of Electrical and Electronic Engineering, The \\ University of Nottingham, NG7 2RD, UK \\ ${ }^{d}$ The Composites Research Group, The University of Nottingham, Nottingham, NG7 2RD, UK
}

\begin{abstract}
This paper reports results on the shielding effectiveness parameter of laminated epoxy composites with carbon fibre reinforcements. Measurements of shielding effectiveness were carried out with a coaxial transmission line testing chamber according to ASTM 4935 standard and epoxy-matrix composites with continuous carbon-fibres were proven to be an excellent electromagnetic interference shielding material, where a composite slab made of 4 layers of prepregs provided more than $99.9 \%$ of electromagnetic attenuation. It was found that the reflection mechanism of the shielding material was mainly influenced by the fibre volume ratio, and that an increase in the number of layers of the composite resulted in higher shielding effectiveness due to a greater absorption mechanism. Calculations of the shielding effectiveness parameter of the material used were made by means of commercial electromagnetic simulation tools, having determined experimentally the overall resistivity of the composite. The findings presented in this work suggest that in presence of a greater number of interfaces at different impedance the separate modelling of matrix and fibres at mesoscopic scale must be taken into account.
\end{abstract} Keywords: Carbon-fibre composites, Polymer-matrix composites, Shielding effectiveness, Electromagnetic interference

\section{Introduction}

Nowadays, the rapid development of electronic technology and the requirement for high-speed electronic systems and devices are leading to an increasing improvement of the performances of electronic and telecommunication devices, such as mobile phones, television, wireless systems, navigation systems and radar [1]. A large field of application areas range from commercial and scientific electronic

\footnotetext{
*Corresponding author.

Email address: Daniela.Munalli@nottingham.ac.uk (D. Munalli)
} 
instruments used in our everyday life to aerospace systems and military electronic products [2]. The advances made have enhanced the quality level of human lives, but at the same time the resulting increases in electromagnetic radiation emitted has raised the question of electromagnetic pollution. As a consequence, several studies have been presented on the subject of radiative damage caused by electromagnetic waves on living species [3-5]. In addition, the electronic devices themselves are densely integrated and operate at low voltage levels and so may be affected by unwanted electromagnetic fields, with consequent perturbation of their performances [6]. Typical examples includes distortion of radio/TV signals, difficulties in personal communication devices, such as mobile phones, limitations or impediments of electrical sensitive instruments [7]. The disturbance generated by an external radiation source, which affects electrical devices by electromagnetic induction, electrostatic coupling, or conduction, is called Electromagnetic Interference (EMI) [8]. Protecting these devices from incoming EMI becomes important to preserve their integrity and functionality.

To address the problem of EMI and electromagnetic pollution, suitable mechanisms of shielding from radiation are needed. EMI shielding is defined as the act of controlling electromagnetic interference by blocking the field radiation with barriers made of conductive or magnetic materials [9]. Shielding materials present the ability to obstruct and minimize the signal emitted through backwards reflection of the field waves or through absorption and dissipation of the radiation power inside the material [10]. In order to reflect the electromagnetic radiation, the shield must be characterized by mobile charge carriers and therefore tends to be electrically conducting; however, high conductivity is not required and a volume resistivity of $1 \Omega \mathrm{cm}$ is accepted [11]. Thus, traditional shielding materials consist of sheet metal, metal screen, meshes, metal foam and volume conductive paints with metal powders [11]. To quantify how much these materials exhibit shielding potential, the Shielding Effectiveness (SE) parameter is widely used. For metals the SE can be calculated through the Schelkunoff's equations, knowing the magnetic permeability and electric conductivity [12, 13]. This index is expressed in $\mathrm{dB}$ and for most industrial and consumer applications a signal reduction of 30 $\mathrm{dB}$ is considered adequate [14], for the making of electronic housing ad for electronic appliances at least $40 \mathrm{~dB}$ are required $[15,16]$, a SE value higher than $100 \mathrm{~dB}$ implies that the material is essentially impenetrable.

Over recent years, alternatives to traditional metals are being studied for possible EMI shielding applications. In particular, various research has been carried out on polymer based conductive composites and their application to the enclosures of electronic devices. The advantages offered by conductive plastic composites over conventional metals include lighter weight, corrosion resistance, lightning protection, and flexibility in design, which abates the housing seams typical of a metallic sheets shield [17, 18]. Polymer matrix composites with carbon based filler have therefore been studied as a promising alternative, especially those with carbon nanotubes [19-23]. However, in the aeronautic 
industry Carbon Fibre Reinforced Polymers (CFRP) are extensively used and show also good shielding properties combined with their typical characteristics of high mechanical resistance (high modulus of elasticity and low density) and high chemical inertness. In this class of composites the shielding efficiency depends on the conductive filler used as the the polymeric matrix is non conductive, and they possess a mean conductivity of $\simeq 10^{4} \mathrm{Sm}^{-1}$, three orders lower than metals [24]. To obtain an high level of shielding effectiveness the filler should have small size unit, high conductivity and high aspect ratio, to increase the electrical connectivity [11]. Extensive studies have been made on the shielding effectiveness of carbon materials including carbon fibres, colloidal graphite and flexible graphite by Chung [19, 20, 25, 26]. Li and Chung [26] compared the SE of polyethersulfone (PES)-matrix composites filled with isotropic pitch based carbon fibres at different percentage of volume filler and different filament lengths, it was found that the SE of composites increased with longer filaments, and that for a $\mathrm{SE}>50 \mathrm{~dB}$ the minimum filler volume fraction was 40\%. Luo and Chung [19] further demonstrated that the SE of polymer-matrix composites with continuous carbon-fibres was greater than the SE provided by polymer with discontinuous filler and showed that the dominant mechanism of EMI shielding was reflection. Wu and Chung [20] used the activation method on carbon fibres to enhance the SE ability. The non linear behaviour of the SE as function of the frequency for CFRP sheet with $10 \%$ of fibre concentration was explained as a consequence of the permittivity complex form of the material by Fouladgar et al. [27]. Several studies investigated the connection between SE and number of layers, fibre concentration and aspect ratio. An increase in the number of layers of the composite was demonstrated to be connected to an higher SE, while a decrease of filler concentration was linked to a shift towards higher frequencies of the maximum SE, where the SE was found to be independent from the fibre volume ratio for frequencies greater than $8 \mathrm{~dB}$ [27, 28]. A higher EMI shielding was related to a greater fibre concentration due to an increase in the absorption loss, and a similar behaviour was observed also for an increased fibre aspect ratio and sample thickness [26, 28-31].

For composite materials the calculation of shielding effectiveness at the design development stage can be realized with 3D full-wave numerical simulations. Simulation tools, such as ANSYS HFSS, EZFDTD and CST Microwave Studio (as utilised in this work), can be used and require electromagnetic parameters of the materials to be known [32,33]. However, for non-metal materials the SE cannot be calculated and is obtained experimentally, with equipment defined by standard methods, such as the MIL-STD-285 and ASTM D4935 standard tests. The equipment required may consist of rectangular or coaxial waveguides, or in other configurations, spot-focusing horn lens antennas [34, 35]. CST Microwave Studio can be used for the design of the waveguides and the antennas described by the standard measurement tests, or for the simulation of incident plane waves and consequently can be applied for calculations of SE [36-38]. Previous work related to the use of CST for SE predictions includes mostly calculation of SE for metals, such as aluminium and steel [36], aluminium rectangular 
enclosures with different apertures [39, 40], and the modelling of new enclosure configurations [41, 42]. However, recently simulations to assess the applicability of composite materials to electromagnetic wave shielding enclosures have been studied [43, 44].

The principal novelty of this work concerns the extension of the EMI shielding theory for a conductive plate to the case of woven carbon fibre/epoxy composite laminates over the percolation threshold. A standardized method involving a flanged coaxial transmission-line holder was used to successfully assess the conductivity/resistivity of thin woven laminates, using experimental measures of their shielding effectiveness. The analysed composites contained continuous carbon fibres reinforcements of high strength intertwined according to a $2 / 2$ twill weave pattern, which could be used in general engineering, robotics and aerospace applications. It was shown that the numerical electromagnetic simulations could successfully predict the SE of composite materials with homogenized electric properties for small thicknesses $\simeq 1 \div 3 \mathrm{~mm}$, without taking into account the mesoscopic characterization of the material. In addition, the SE of dry fibres intertwined with the same pattern as the composite laminates was measured. The work proved that one layer alone of dry woven fibers was able to shield more than $99 \%$ of the electromagnetic radiation, where the predominant shielding mechanism was due to reflection loss.

This paper is organized as follows. In section 2, the aspects of the EMI shielding theory are reviewed, including the shielding mechanism and principles behind SE measurements techniques. Then, in section 3 experimental data obtained with a coaxial line assembly are analysed for carbon fibre/epoxy plates and for dry carbon fibres. Finally, simulations of the experimental equipment are presented in section 4, where the composites are simulated as homogeneous elements with homogenized electric properties.

\section{EMI shielding theory}

\subsection{The shielding mechanism}

The electromagnetic plane wave theory is applied for EMI shielding in the far field region where the shield distance from the radiation source is larger than $\lambda / 2 \pi$, being $\lambda$ the free space wavelength of the electromagnetic field [45].

According to Schelkunoff's approach when an electromagnetic plane wave is incident on a shielding material a part of the wave energy is reflected at the interface with the shield, as a result of the difference in impedance between the incident wave and the shield $[8,46]$. This primary mechanism, defined as reflection loss, requires the presence of charge carriers in the material, i.e. for the shield to be electrically conducting, and is a function of the ratio between the electrical conductivity and the magnetic permeability of the shield $\sigma / \mu[7]$. A part of the wave energy is then absorbed by the volume of the material, this second mechanism is called absorption loss and is inversely proportional 
to the penetration depth or skin depth of the material, defined for high loss materials by the equation $[8,12,13]$ :

$$
\delta=\frac{1}{\sqrt{\pi f \mu \sigma}}
$$

where $\delta$ is the depth of the material at which the electromagnetic field drops exponentially to $1 / e$ of the incident value and $f$ is the frequency. The absorption loss is the result of the heat generated in the shield and is related to the interaction of electric and/or magnetic dipoles with the electromagnetic field.

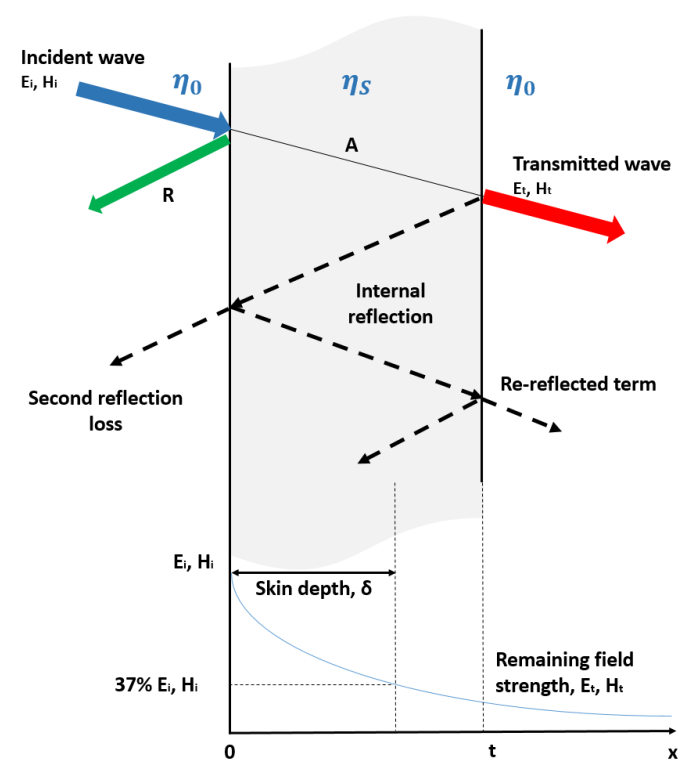

Fig. 1: Concept of shielding effectiveness (SE) for a slab of conductive material, wave absorption, reflection, re-reflection and transmission mechanisms.

In addition a third part of the electromagnetic radiation is attenuated by multiple reflections, which take place at various surfaces or interfaces in the shield, this third mechanism can be disregarded for SE calculations if the shield thickness is much greater than the skin depth [46], when the absorption loss is $\geq 15 \mathrm{~dB}$ [22]. Finally the last part of the electromagnetic field is transmitted through the material, so that the effectiveness of the shield can be viewed as the ratio expressed in $\mathrm{dB}$ of the electric (magnetic) incident field intensity to the intensity of the electric (magnetic) field that is transmitted through the shield:

$$
S E=20 \log _{10}\left(\frac{E_{i}}{E_{t}}\right)=20 \log _{10}\left(\frac{H_{i}}{H_{t}}\right)=10 \log _{10}\left(\frac{P_{i}}{P_{t}}\right)
$$

where the subscripts ' $i$ ' and ' $t$ ' indicate respectively the incident radiation and the transmitted radiation. Alternatively the expression provided by the ratio of the powers of the incident and transmitted electromagnetic signals can also be used. 


\subsection{EMI SE of a conductive plate}

The shielding effectiveness can thus be expressed as the sum of three contributions [13, 47, 48]:

$$
S E=S E_{R}+S E_{A}+S E_{M R}
$$

Each contribution, reflection loss $\left(S E_{R}\right)$, absorption loss $\left(S E_{A}\right)$ and multiple reflection $\left(S E_{M R}\right)$, influence the overall ability of a polymer composite to shield from radiation. In order to have a better understanding of these three mechanisms, the case study of a homogeneous conductive slab will be presented. As shown in Fig. 1 the electromagnetic wave propagates in the free space until it encounters a material with different intrinsic impedance $\eta_{S}$. In the far field region the intrinsic impedance of the free space is given by the ratio between the incident electric field amplitude and the incident magnetic field amplitude and is a constant equal to $377 \Omega$ :

$$
\eta_{0}=\frac{\left|\boldsymbol{E}_{\boldsymbol{i}}\right|}{\left|\boldsymbol{H}_{\boldsymbol{i}}\right|}=\sqrt{\frac{\mu_{0}}{\varepsilon_{0}}} \simeq 377 \Omega
$$

while the corresponding intrinsic impedance of a good conductor is [13]:

$$
\eta_{S}=\sqrt{\frac{j \omega \mu}{\sigma+j \omega \varepsilon}} \simeq \sqrt{\frac{j \omega \mu}{\sigma}}
$$

where, given the hypothesis of a good conductor with high conductivity, $\sigma \gg \omega \varepsilon$. The reflection loss parameter is related to this difference in characteristic impedances.

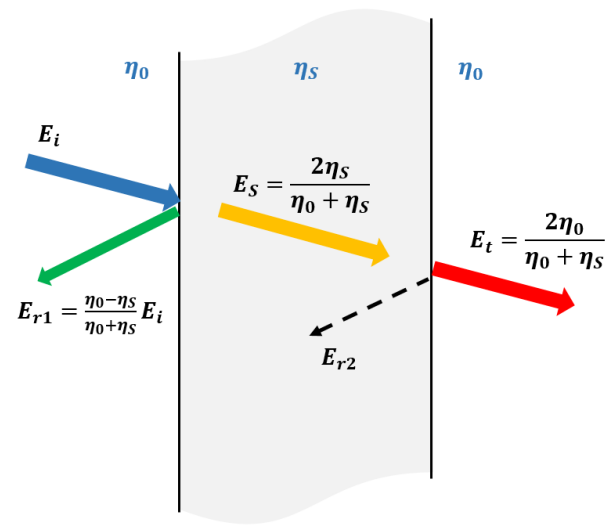

Fig. 2: Incident wave transmitted at two boundaries with different impedance. $E_{S}$ is the transmitted wave at the first interface and $E_{r}$ is the reflected wave [8].

As shown in Fig. 2 the intensity of the transmitted electric field at the first interface is:

$$
E_{S}=\frac{2 \eta_{S}}{\eta_{0}+\eta_{S}} E_{i}
$$


At the second boundary of the shield, the wave passes from a medium with impedance $\eta_{S}$ to the free space:

$$
E_{t}=\frac{2 \eta_{0}}{\eta_{0}+\eta_{S}} E_{S}=\frac{4 \eta_{0} \eta_{S}}{\left(\eta_{0}+\eta_{S}\right)^{2}} E_{i}
$$

Substituting the wave impedance prior to entering the shield $\eta_{w}$ for $\eta_{0}$ and disregarding the effect of the multiple reflections $S E_{R}$ can be defined as $[8,13]$ :

$$
S E_{R}=20 \log _{10} \frac{E_{i}}{E_{t}}=20 \log _{10} \frac{\left(\eta_{w}+\eta_{S}\right)^{2}}{4 \eta_{w} \eta_{S}}
$$

If the shield is an homogeneous conductive plate surrounded by an insulator, then the impedance of the shield is much smaller than the impedance of the incident wave and the largest reflection occurs at the first boundary encountered by the incident electric field, so also a thin sheet of conductive material can provide a good reflection loss. Therefore if $\eta_{w} \gg \eta_{S}[8]$ :

$$
S E_{R}=20 \log _{10} \frac{\eta_{w}}{4 \eta_{S}}
$$

The absorption loss $S E_{A}$ parameter is related to the decrease of the electromagnetic field amplitude. On encountering a shield made of a conductive material, the amplitude of the electromagnetic wave decreases exponentially, according to equation:

$$
E_{t}=E_{i} \mathrm{e}^{-t / \delta}
$$

where $t$ is the thickness of the shield and $\delta$ the skin depth. The absorption loss can therefore be written as $[8,13]$ :

$$
S E_{A}=20 \log _{10} \frac{E_{i}}{E_{t}}=20 \log _{10}\left(e^{\frac{t}{\delta}}\right)=20\left(\frac{t}{\delta}\right) \log _{10}(e)
$$

Substituting Eq. (1) into Eq. (11):

$$
\left.S E_{A}\right|_{d B} \simeq 15.4 t \sqrt{f \mu \sigma}
$$

where Eq. (12) shows the dependence of the absorption loss on the square root of the product of the permeability and conductivity of the shield material. Thus the absorption loss increases with frequency and the thickness of the shield.

Finally the third contribution to the SE for a slab of conductive material surrounded by an insulating medium is linked to the primary reflection of the magnetic field. While for the electric field the primary reflection occurs at the first interface, for the magnetic field the primary reflection occurs at the second boundary of the shield and multiple reflections generates within the shield. If the shield is thin and the absorption loss is not significant, the reflected wave at the second boundary returns to the first boundary where is reflected again and so on until the amplitude of the field gradually decreases 
because of the multiple passages through the thickness of the shield. Therefore this correction factor due to the multiple reflections related to the propagation of the magnetic fields in a shield is $[8,13]$ :

$$
S E_{M R}=20 \log _{10}\left|1-\frac{\left(\eta_{S}-\eta_{w}\right)^{2}}{\left(\eta_{S}+\eta_{w}\right)^{2}} e^{-2 t / \delta}\right|
$$

$S E_{M R}$ is a function of the ratio $t / \delta$ and is a negative number. This parameter can be neglected if the thickness of the shield is greater than the skin depth, for plane waves the reflection loss is high and the re-reflection loss is small. If the thickness of the material is equal to the skin depth the correction factor is less than $2 \mathrm{~dB}$, when $t=0.1 \delta$ the correction factor is $15 \mathrm{~dB}$ [8].

\subsection{EMI SE measurement of non metallic materials}

A two port vector network analyser (VNA) connected across a coaxial or rectangular waveguide sample holder can be used for measuring the power reflected $P_{r}$ and transmitted $P_{t}$ at the two ports, when the incident power is known $P_{i}[48,49] . P_{i}$ can be defined as the sum of three terms, power reflected towards port 1 at the first boundary air/composite, power absorbed and power transmitted after the second boundary composite/air:

$$
P_{i}=P_{r}+P_{a}+P_{t}
$$

The values of the SE parameters are obtained by means of the phase and magnitude of the scattering parameters $S_{11}$ (or $S_{22}$ ) and $S_{21}$ (or $S_{12}$ ), where they represent the reflectance (R), the transmittance (T) and the absorbance (A) [48, 49]:

$$
\begin{gathered}
R=\left|S_{11}\right|^{2}=\frac{P_{r}}{P_{i}} \\
T=\left|S_{21}\right|^{2}=\frac{P_{t}}{P_{i}} \\
A=\frac{P_{a}}{P_{i}}=1-R-T
\end{gathered}
$$

Considering the power balance, the SE is given by the sum of the shielding by reflection and the shielding by absorption contributions, in case of negligible $S E_{M R}, S E_{R}$ and $S E_{A}$ can be expressed in mathematical terms as follows [45]:

$$
\begin{aligned}
S E & =S E_{R}+S E_{A}= \\
& =10 \log _{10} \frac{P_{i}}{P_{t}}= \\
& =10 \log _{10} \frac{P_{i}}{P_{i}-P_{r}}+10 \log _{10} \frac{P_{i}-P_{r}}{P_{t}}= \\
& =10 \log _{10} \frac{1}{1-\left|S_{11}\right|^{2}}+10 \log _{10} \frac{1-\left|S_{11}\right|^{2}}{\left|S_{21}\right|^{2}}= \\
& =10 \log _{10}\left|\frac{1}{\left|S_{21}\right|^{2}}\right|
\end{aligned}
$$


Therefore the measure of the transmission coefficient $S_{21}$, i.e. the forward voltage gain, gives the value of the overall SE along the range of frequencies considered, according to Eq. (18).

\subsection{SE as function of the resistivity}

When Eq. (9) and Eq. (12) are substituted in Eq. (3), having disregarded the multiple reflections mechanism, the following expression is obtained:

$$
S E=20 \log _{10} \frac{\eta_{w}}{4 \eta_{S}}+15.4 t \sqrt{f \mu \sigma}
$$

Combining Eq. (5) with Eq. (19) and considering that a polymer composite material is non-magnetic and therefore $\mu=\mu_{0}$, the SE becomes:

$$
S E=20 \log _{10} \eta_{w}-20 \log _{10}\left(4 \sqrt{\frac{\omega \mu_{0}}{\sigma}}\right)+15.4 t \sqrt{f \mu_{0} \sigma}
$$

Thus it is possible to express the EMI shielding of conductive plastics, such as electroconductive composites, as a function of the resistivity (or conductivity) of the composite material, the frequency and the thickness of the shield. This expression is called Simon formalism [50, 51]:

$$
\left.S E\right|_{d B}=50+10 \log _{10}(\rho f)^{-1}+1.7 t(f / \rho)^{1 / 2}
$$

where $f$ is the frequency in $\mathrm{MHz}, t$ the thickness of the material in $\mathrm{cm}$ and $\rho$ is the volume resistivity expressed in $\Omega \mathrm{cm}$. The reflection mechanism $S E_{R}$ is estimated by the first two terms, the third term evaluates the absorption loss $S E_{A}$.

\section{Experimental Evaluation of SE}

\subsection{Materials}

EMI shielding measurements were performed on carbon fibre/epoxy composites and dry carbon fibres. Two carbon fibre/epoxy laminates samples were prepared using pre-impregnated fabrics of two different weights, while two specimens were realized with only layers of dry carbon reinforcements in order to verify the impact on the SE parameter of the absence of the epoxy resin medium (Fig. 3). To observe meaningful results the dry carbon fibers reinforcements considered are of the same type and same pattern as the ones present in the prepregs.

The high strength carbon fibre laminates were made of layered prepregs: XPREG XC130 210gsm and XPREG XC130 450gsm, with standard carbon fibre reinforcements Pyrofil TR30S 210gsm 3k and TR50S 450gsm $12 \mathrm{k}$ respectively, intertwined according to a $2 / 2$ twill weave pattern. The stacking sequence and thicknesses are reported in Table 1 . Each layer is orientated in a $0^{\circ}$ and $90^{\circ}$ orientation. There was no layer rotation during the lay up procedure. After layering the prepregs as per Table 

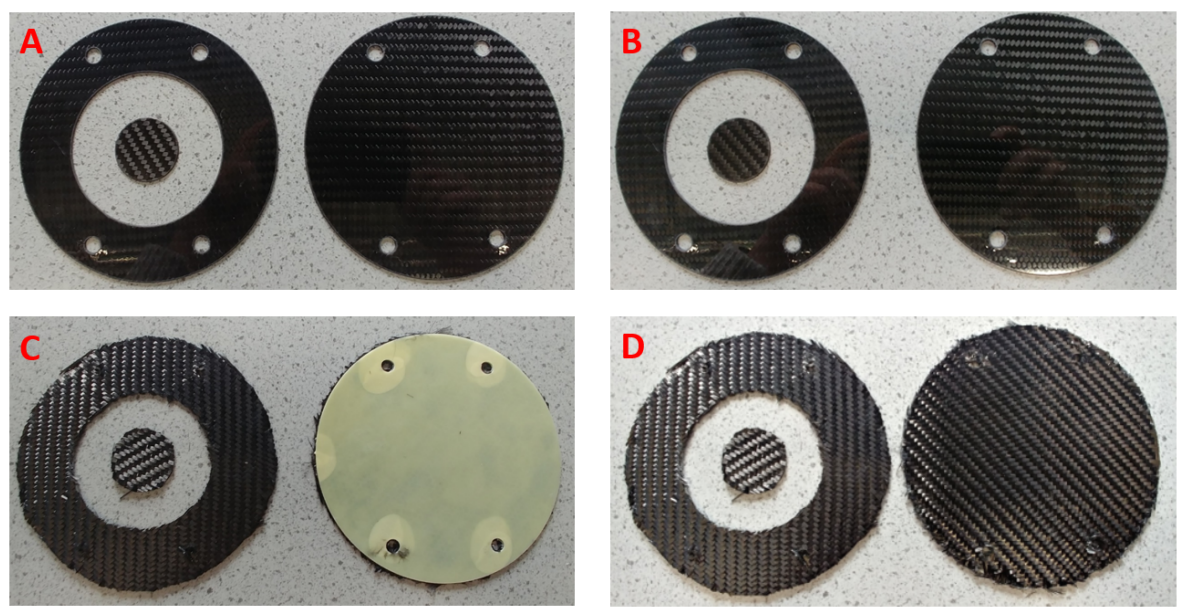

Fig. 3: A) Specimen A: CFRP laminate (XPREG XC130-210gsm/XPREG XC130-450gsm/XPREG XC130450gsm/XPREG XC130-210gsm); B) Specimen B: CFRP laminate (XPREG XC130-210gsm/3xXPREG XC130-450gsm/XPREG XC130-210gsm/2xXPREG XC130-450gsm/XPREG XC130-210gsm); C) Specimen C: 1 layer of dry fibres (TR30S); D) Specimen D: 4 layers of dry fibres (TR30S/TR50S/TR50S/TR30S).

1 , both the wet samples underwent a curing cycle in autoclave at $100^{\circ} \mathrm{C}$ and pressure at 8 bar under vacuum for 4 hours. Laminates of $1 \mathrm{~mm}$ thickness (specimen A) and $3 \mathrm{~mm}$ thickness (specimen B) were obtained.

Table 1: Carbon fibres/epoxy laminates: Specimen A and B properties

\begin{tabular}{|c|c|c|c|}
\hline Specimen & Thickness (mm) & No of plies & Stacking sequence \\
\hline \multirow[t]{4}{*}{ A } & $1 \pm 0.15$ & 4 & XPREG XC130-210gsm \\
\hline & & & XPREG XC130-450gsm \\
\hline & & & XPREG XC130-450gsm \\
\hline & & & XPREG XC130-210gsm \\
\hline \multirow[t]{8}{*}{ B } & $3 \pm 0.25$ & 8 & XPREG XC130-210gsm \\
\hline & & & XPREG XC130-450gsm \\
\hline & & & XPREG XC130-450gsm \\
\hline & & & XPREG XC130-450gsm \\
\hline & & & XPREG XC130-210gsm \\
\hline & & & XPREG XC130-450gsm \\
\hline & & & XPREG XC130-450gsm \\
\hline & & & XPREG XC130-210gsm \\
\hline
\end{tabular}

Subsequently, in order to analyse the impact of the carbon reinforcements only, samples with 
respectively one layer of dry fibres (specimen C) and with four layers of dry fibres (specimen D) were prepared. The stacking sequence can be found in Table 2 .

Table 2: Dry carbon fibres fabrics: Specimen C and D properties

\begin{tabular}{lc}
\hline Specimen & Dry fibres Layers \\
\hline C & TR30S \\
D & TR30S/TR50S/TR50S/TR30S \\
\hline
\end{tabular}

Specimen $\mathrm{C}$ was made by gluing one layer of dry carbon fibres TR30S, orientated according to $0^{\circ}$ and $90^{\circ}$ orientation, on a fibreglass panel which displays a good dielectric behaviour and is transparent to radio waves. Specimen $\mathrm{C}$ was made with the aim to assess the SE of only one layer of dry fibres.

The same procedure was applied to specimen D, where instead four layers of dry fabric, orientated according to $0^{\circ}$ and $90^{\circ}$ orientation each, were glued to a fiberglass support in order to mimic the stacking sequence of Specimen A and to observe the effect on the overall SE of the absence of the polymer matrix which is a common electrical insulator with average electrical resistivity of the order of $\simeq 10^{14}: 10^{16} \Omega \mathrm{m}$ [52]. Specimen A can therefore be compared to specimen D and an increase in shielding effectiveness is to be expected as a result of the direct contact between the carbon fibres. The experimental tests confirmed this behaviour to the point where specimen D exceeded the dynamic range of the Network Analyser; hence, the preparation of a fifth specimen, which was repeating the stacking sequence of specimen B, was considered not useful for the study purposes, because of the greater shielding power provided by doubling the number of layers of dry carbon fibers and the consequent lack of measurable results.

Finally, the samples were shaped by waterjet cutting with the dimensions reported in Fig. 4. For each material to be tested a reference specimen and a load specimen were prepared, the reference is composed by two parts having dimensions equal to the inner and outer conductors of the coaxial line, while the load has the diameter equal to that of the outer conductor of the coaxial line.
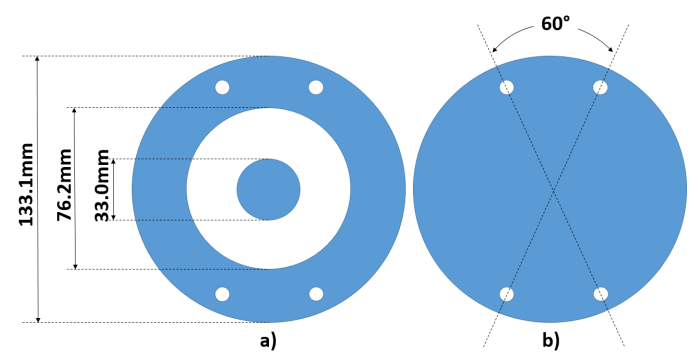

Fig. 4: ASTM D4935: a) Reference specimen; b) Load specimen. 


\subsection{ASTM D4935 method}

The experimental measurements were realized by means of a coaxial transmission line, according to the ASTM D4935 standard, used to measure the shielding effectiveness of planar specimens [34]. The advantages offered by this test technique are repeatability of measurements and the possibility to determine the components of the electromagnetic signal, i.e. which part of the signal is reflected, absorbed or transmitted [2]. The set-up as illustrated in Fig. 5 consisted of a programmable E5062A ENA-L RF network analyser coupled with a coaxial specimen holder unit realized with an external and internal conductors made of brass, the outer conductor is expanded to maintain throughout the entire length of the holder a $50 \Omega$ impedance and $10 \mathrm{~dB}$ attenuators are placed at each end of the specimen holder with the function of insulators from the input and output signal.

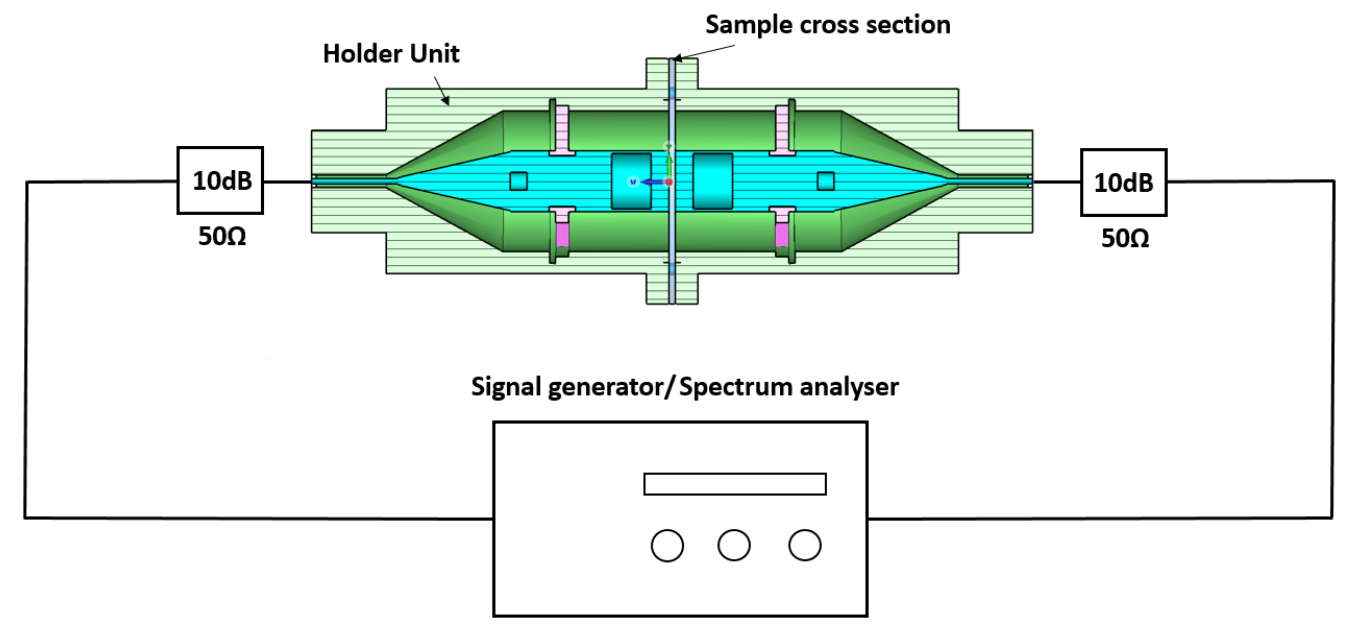

Fig. 5: Set-up for electromagnetic shielding effectiveness measurement.

The inferior limit of the dynamic measurement range was ensured using a standard metallic attenuator with a maximum attenuation of $\simeq 110 \mathrm{~dB}$; for the measurements, values within $10 \mathrm{~dB}$ of the smallest detectable signal were considered beyond the dynamic range (DR) according to the ASTM D4935 standard. The frequency was scanned from $30 \mathrm{MHz}$ to $1.5 \mathrm{GHz}$ to ensure TEM wave radiation, and the values of the incident power (voltage) and the transmitted power (voltage) through the shield were recorded in 401 data points at each frequency. Two rounds of measurements were required for each material. The SE parameter was calculated measuring first the received power while using the reference specimen $P_{t}^{R e f}$, this allows to repeat the capacitive coupling of the loaded case, when establishing the reference level, leaving the space between the two conductors free (unloaded). Once replaced the reference with the load specimen, the test was repeated to measure the received power 
$P_{t}^{\text {Load }}$. Therefore according to Eqs. $(16,18)$ the SE is given by:

$$
\left.S E\right|_{d B}=\left|S_{21}^{\text {Load }}\right|_{d B}-\left|S_{21}^{\text {Ref }}\right|_{d B}=10 \log _{10} \frac{P_{t}^{\text {Load }}}{P_{i}}-10 \log _{10} \frac{P_{t}^{\text {Ref }}}{P_{i}}=10 \log _{10} \frac{P_{t}^{\text {Load }}}{P_{t}^{\text {Ref }}}
$$

Furthermore, the power reflected back to the source and the power transmitted to port 2, for both reference and load specimens are obtained from the values of the scattering parameters $S_{21}$ and $S_{11}$ :

$$
\begin{aligned}
& P_{r}^{\text {Ref } / \text { Load }}=P_{i} 10 \frac{S_{11}^{\text {Ref } / \text { Load }}}{10} \\
& P_{t}^{\text {Ref/Load }}=P_{i} 10^{\frac{S_{21}^{\text {Ref } / \text { Load }}}{10}}
\end{aligned}
$$

\subsection{Experimental results}

The measurements were recorded using a receiver bandwidth of $300 \mathrm{~Hz}$ and a signal power of 5 $\mathrm{dBm}$, to lower the minimum range detectable and reduce the signal to noise ratio. Fig. 6 shows the scattering parameter $S_{21}$ for specimen A, B and C, both in case of references and loads. For the carbon fibres/epoxy laminates (samples A and B) it was possible to obtain SE values for a range of frequencies up to $450 \mathrm{MHz}$, beyond this point the measured values exceed the DR of the system. This behaviour is caused by the low resistivity of carbon fibres that makes the composite acting like a conductor. On the contrary SE values for 1 layer of dry carbon fibres were registered for all the range of frequencies from $30 \mathrm{MHz}$ to $1.5 \mathrm{GHz}$, being a single woven fabric of dry fibres less conductive and consequently less effective as a shield than layered composites as samples A and B.

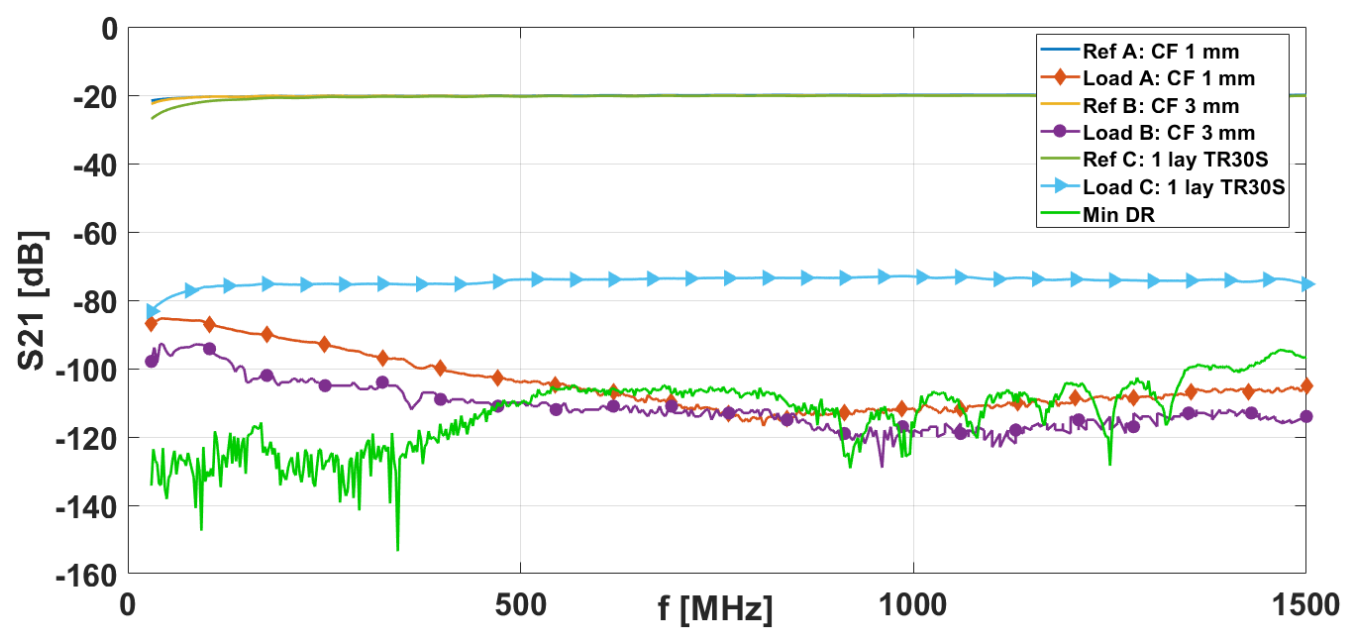

Fig. 6: S-parameter $S_{21}$ of carbon fibres-epoxy laminate $1 \mathrm{~mm}$ (specimen A), carbon fibres-epoxy laminate 3mm (specimen B), 1 layer of dry carbon fibres TR30S (specimen C).

However, the situation changes when comparing 4 layers of dry fibres (sample D) with sample A. Both samples have the same stacking sequence of woven fabrics, but specimen D shows greater 
shielding power than specimen A, as it appears in Fig. 7, where $S_{21}$ decreases in dB towards the lower measurement limit, exceeding the DR, therefore the presence of epoxy resin lessens the contact points between fibres, reducing the overall conductivity of the composite.

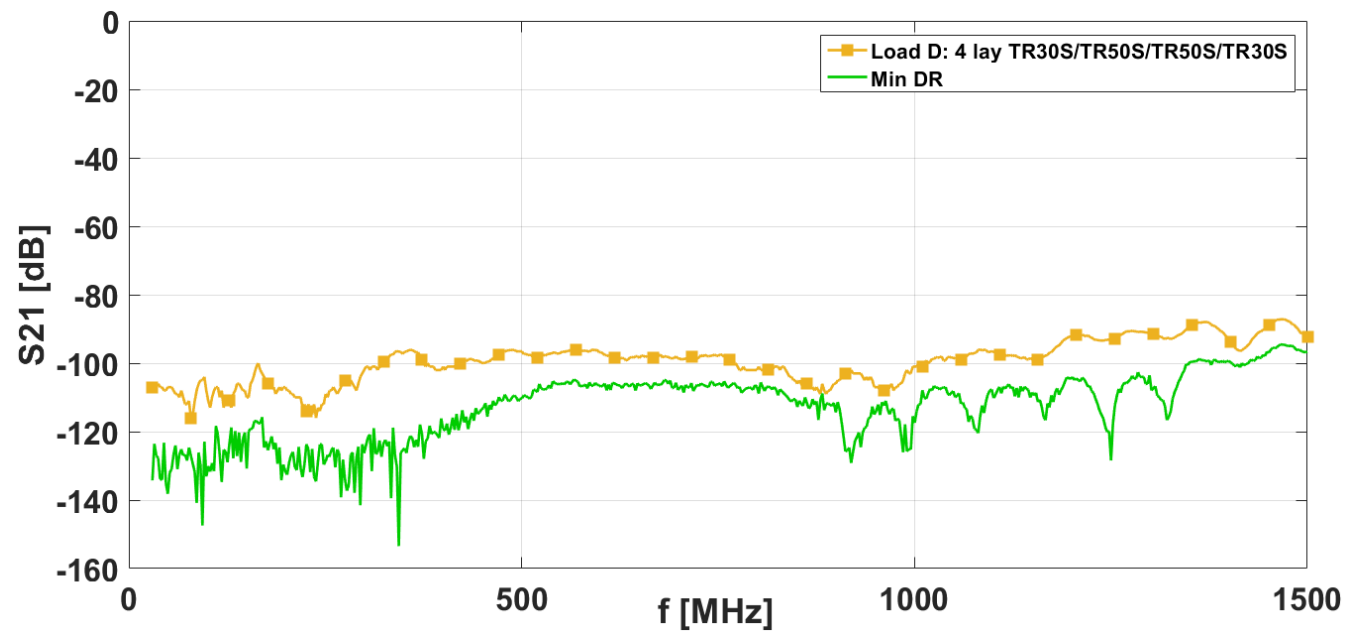

Fig. 7: S-parameter $S_{21}$ of specimen D: 4 layers of dry carbon fibres (TR30S/TR50S/TR50S/TR30S).

Table 3 shows the values of the average volume resistivities and of the average SE for the tested samples. Sample B (8 layers of fabric prepregs) exhibits higher attenuation upon transmission compared to sample A (4 layers of fabric prepregs). As a rule of thumb the greater the resistivity (smaller

Table 3: Electrical volume resistivity and attenuation upon transmission of samples A, B and C

\begin{tabular}{lllccc}
\hline Specimen & Matrix & Filler & $\begin{array}{c}\text { Frequency range } \\
(\mathbf{M H z})\end{array}$ & $\begin{array}{c}\text { Average volume } \\
\text { resistivity }(\boldsymbol{\Omega c m})\end{array}$ & $\begin{array}{c}\text { Average } \\
\text { SE (dB) }\end{array}$ \\
\hline A & Epoxy & Carbon fibres & 30 to 450 & $9.8 \times 10^{-3}$ & $72.8 \pm 5.5$ \\
B & Epoxy & Carbon fibres & 30 to 400 & $3.3 \times 10^{-2}$ & $81.6 \pm 5.3$ \\
C & / & Carbon fibres & 30 to 1500 & $/$ & $53.9 \pm 0.6$ \\
\hline
\end{tabular}

conductivity), the lower the shielding effectiveness, because of the lower reflection loss coefficient $S E_{R}$ $[7,19,20]$. However, the volume resistivities calculated with Simon formalism, Eq. (21), using the SE measured values and the measurement frequencies, prove that sample B has also a higher resistivity (lower conductivity) than sample A and that consequently a great contribution to the SE is given by the absorption loss $S E_{A}$ which is directly proportional to the thickness of the specimen, in fact the higher shielding effectiveness of sample B according to Eq. (21) is caused by a greater absorption loss, due to the greater thickness of the specimen. The volume fraction of polymeric matrix is possibly bigger in specimen B compared to specimen A, also the larger thickness and the different lay up (the 
presence of 5 layers of XPREG XC130 450g) may have influenced the spillage of the resin during the curing process in autoclave. A largest amount of resin reduces the overall electrical conductivity of the material because it increases the fraction of insulating material and decreases the contact points between the conductive fibres.

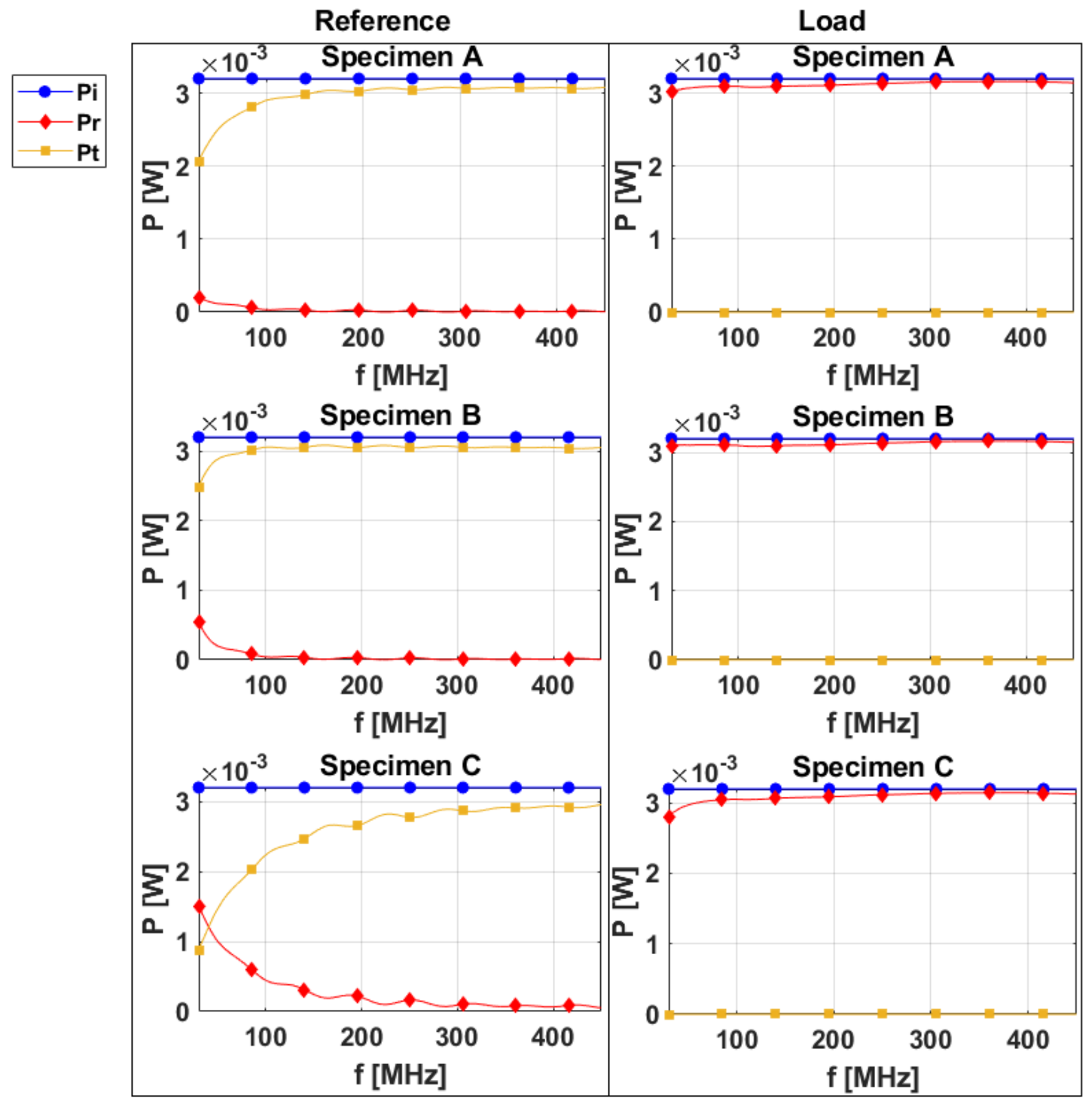

Fig. 8: Incident, reflected and transmitted powers for Specimen A/B/C. The first column refers to Reference specimens in place, second column refers to Load specimens in place.

Fig. 8 shows the incident, reflected and transmitted power of the three different specimens as a function of the frequency. In the first column the reported power values were measured with the reference in place, while in the second column the load is considered. When the reference is in place, 
the power transmitted tends to $P_{i}=0.0032 \mathrm{~W}$, but it doesn't reach its value, because realistically the contact between flanges and specimen is not perfect and a discontinuity is introduced, which leads to power losses. In addition, a certain amount of power reflected could be measured for specimen $\mathrm{A}, \mathrm{B}$ and $\mathrm{C}$ of the order of $10^{-5} \div 10^{-6} \mathrm{~W}$, representing the signal reflected from the coaxial holder system. In case of loaded transmission line, the situation is reversed. The power transmitted to port 2 is extremely low of the order of $10^{-10} \mathrm{~W}$ for specimen $\mathrm{A}, 10^{-12} \mathrm{~W}$ for specimen $\mathrm{B}$ and $10^{-8} \mathrm{~W}$ for specimen A, confirming the high shielding ability of carbon fibres. The power reflected back to port 1 is very high and around $0.0031 \mathrm{~W}$.

Table 4 provides the percentages of the incident power $P_{i}$ that is reflected back to the source and transmitted though the material under test. Considering specimens A and B, it can be observed that, when the reference is in place, the power reflected from the system increases with the thickness of the sample, whilst with the load in place the reflected power (which includes also the contribution of the experimental apparatus) remains almost unchanged; however, at the same time the signal transmitted through the load decreases for specimen B. This behaviour potentially indicates a greater power attenuation due to the absorption loss for specimen B, because of the larger number of layers contained. The primary mechanism of EMI shielding for carbon composites is addressed as reflection

Table 4: Power reflected with load specimen in place, power reflected with reference specimen in place, power transmitted through the load specimen

\begin{tabular}{lccc}
\hline Specimen & $\begin{array}{c}\text { Percentage of power } \\
\text { reflected with load } \\
\text { in place } \\
\left(\boldsymbol{P}_{\boldsymbol{r}}^{\text {Load }} / \boldsymbol{P}_{\boldsymbol{i}}\right)\end{array}$ & $\begin{array}{c}\text { Percentage of power } \\
\text { reflected with reference } \\
\text { in place } \\
\left(\boldsymbol{P}_{\boldsymbol{r}}^{\text {Ref }} / \boldsymbol{P}_{\boldsymbol{i}}\right)\end{array}$ & $\begin{array}{c}\text { Percentage of power } \\
\text { transmitted through } \\
\text { the load } \\
\left(\boldsymbol{P}_{\boldsymbol{t}}^{\text {Load }} / \boldsymbol{P}_{\boldsymbol{i}}\right)\end{array}$ \\
\hline $\mathrm{A}$ & $98.8 \%$ & $8.5 \%$ & $0.017 \%$ \\
$\mathrm{~B}$ & $98.9 \%$ & $9.4 \%$ & $0.004 \%$ \\
$\mathrm{C}$ & $97.6 \%$ & $12.7 \%$ & $0.23 \%$ \\
\hline
\end{tabular}

$[19,53]$; however, the lower amount of power blocked by absorption is caused by the lower power transmitted across the first interface of the shield material because of the previous reflection mechanism.

Considering specimen $\mathrm{C}$, the reflected power with the reference material in place increases, while decreases the signal transmission through the unloaded coaxial holder $(96 \div 97 \%$ of signal transmitted for composites laminates against $94 \%$ for specimen C). A possible explanation to this behaviour is due to the contact between the sample and the flanges. The specimen presents a non smooth surface on one side and a dielectric side, which can potentially exacerbate any issues related to the electrical continuity of the transmission line. The single layer of dry fibres in specimen $\mathrm{C}$ shows an appreciable reflection 
loss comparable with specimen A and B, but also a significant transmission of power. The experimental results demonstrate that the prevailing shielding mechanism of specimen $\mathrm{C}$ is due to return loss and its value is similar to the one of the composite laminates A and B; in fact, the reflection mechanism is highly dependent on the first interface encountered by the electromagnetic waves and both specimens A and B possess as first layer the same woven fabric as specimen C. On the other hand, as expected, the absorption loss is not predominant, due to lack of adequate thickness of the dry fibers layer and consequently a greater part of the radiation passes through the material.

In conclusion, the $\mathrm{SE}$ measured for specimen $\mathrm{C}$ shows that a layer of $2 / 2$ twill weave dry fabric alone is able to shield more than $99.999 \%$ of the electromagnetic radiation. A composite material made of 4 layers of prepregs offers more than $99.99999 \%$ of attenuation, while a composite with 8 layers more than $99.999999 \%$. The higher shielding ability displayed by specimen B can be due to a greater absorption loss; however, an increase in multiple reflections inside the material can also be considered. A material with an attenuation level between $90 \mathrm{~dB}$ and $120 \mathrm{~dB}$ is considered to have an excellent shielding performance [54], in fact any calculated attenuation higher than $100 \mathrm{~dB}$ implies that the material is essentially impenetrable. The composites analysed, having a shielding effectiveness between $60 \mathrm{~dB}$ and $90 \mathrm{~dB}$, provide a significant degree of attenuation of the electromagnetic radiation [54].

\section{Electromagnetic simulations}

CST Studio Suite simulation software was applied to perform calculation of shielding effectiveness, the software is used for designing, simulating and optimizing electromagnetic systems and presents the ability to link multiple simulations with different solvers [36]. The experiment was implemented within the suite's CST Microwave Studio (CST MWS) component, a tool for the 3D electromagnetic simulation of high frequency components such as antennas, filters, couplers, planar and multi-layer structures, where the software offers the possibility to build in the same environment circuit elements and $3 \mathrm{D}$ components [55].

\subsection{Experiment simulation}

The first step of the modelling work was to reproduce the equipment used for the experimental tests, as shown in Fig. 9. The design was realized following the instructions reported in ASTM D4935.

The coaxial line assembly consists of two mirrored parts and is composed of an external conductor and an inner conductor separated by air, built up from several conic and tapered sections. The sample under test sits in the central part of the system, where it is fixed to an outer flange. An acrylic insert provides support for the centre conductor, and it is visible in purple in Fig. 10. The input signal power enters through port 1 and the output power is measured at port 2. In addition air elements 


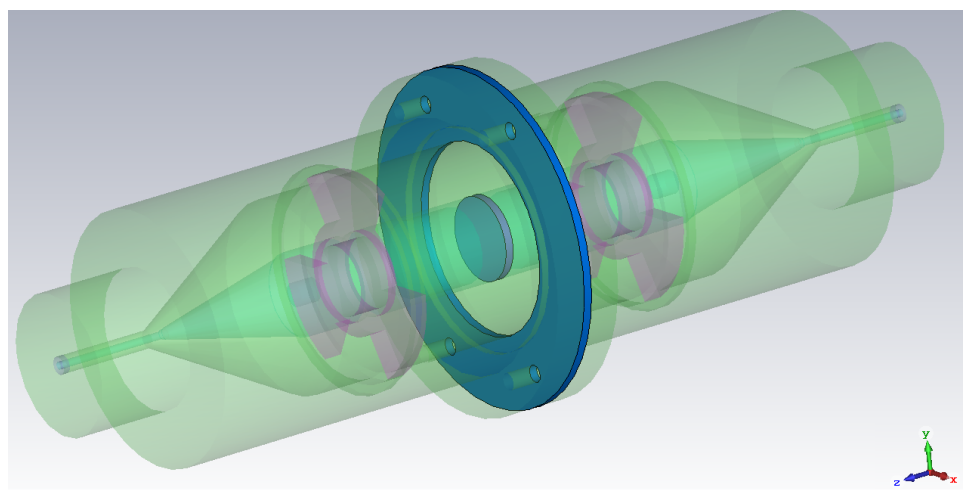

Fig. 9: ASTM D4935 equipment simulation.

were inserted between inner and outer conductors at the ports location to guarantee the passage of electromagnetic radiation.

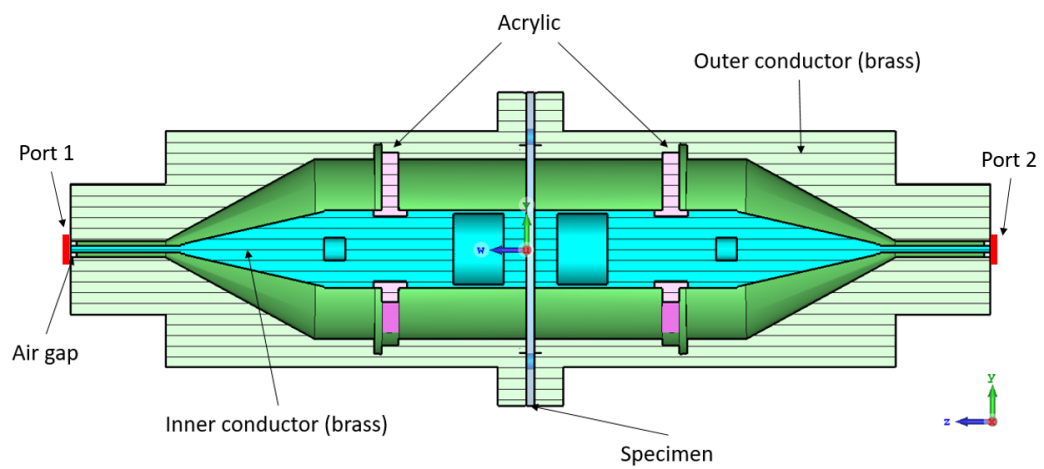

Fig. 10: ASTM D4935 simulation equipment components.

The properties of the selected materials are described in Table 5. The internal and external conductors were made of brass (a lossy metal) with a conductivity of $2.74 \mathrm{e}+007 \mathrm{~S} / \mathrm{m}$, the acrylic material was simulated by means of a material with relative permittivity $\varepsilon=3.2$ and conductivity of $0.02 \mathrm{~S} / \mathrm{m}$. The simulations were carried out for specimen A and specimen B considering one geometry element with homogenized conductivity calculated with Eq. (21) derived by the shielding effectiveness theory.

Table 5: Parameters of the assembly components

\begin{tabular}{lcccc}
\hline Component & Type & $\begin{array}{c}\text { Electrical conductivity } \\
(\mathbf{S} / \mathbf{m})\end{array}$ & $\begin{array}{c}\text { Relative Permittivity } \\
\text { Relative Permeability }\end{array}$ \\
\hline Out/In conductor & Lossy metal & $2.74 \mathrm{e}+07$ & $\boldsymbol{\varepsilon}_{\boldsymbol{r}}$ & $\boldsymbol{\mu}_{\boldsymbol{r}}$ \\
Acrylic & Normal & 0.02 & 3.6 & 1 \\
Air gap & Normal & $/$ & 1.00059 & 1 \\
\hline
\end{tabular}


Once obtained the values of SE as function of the frequency $f$, the conductivities were calculated as the average of the values registered in a range between $30 \mathrm{MHz}$ and $450 \mathrm{MHz}$ for specimen $\mathrm{A}$, and between $30 \mathrm{MHz}$ and $400 \mathrm{MHz}$ for specimen B, using Eqs. (9,12,20). Different thicknesses were considered for both samples A and B, where at the same shielding conditions, the thinnest specimen in order to offer an equal level of protection, has a greater conductivity, as it appears in Table 6. Both cases of reference and load were simulated, obtaining $0 \mathrm{~dB}$ of SE for references, considering no material is actually acting as a shield. The $-20 \mathrm{~dB}$ value constantly measured for the reference specimens during the experimental tests was due to the two $10 \mathrm{~dB}$ attenuators, which are not present in the simulation.

Table 6: Calculated average value of conductivity with Simon formalism for different values of thicknesses

\begin{tabular}{lcc}
\hline Specimen & $\begin{array}{c}\text { Thickness } \\
{[\mathbf{m m}]}\end{array}$ & $\begin{array}{c}\text { Average } \boldsymbol{\sigma} \\
{[\mathbf{S} / \mathbf{m}]}\end{array}$ \\
\hline $\mathrm{A}$ & 1.00 & 10200.65 \\
& 0.85 & 12954.21 \\
$\mathrm{~B}$ & 3.00 & 3161.45 \\
& 2.75 & 3636.28 \\
\hline
\end{tabular}

The electric field lines for the Transverse electromagnetic (TEM) propagation mode are represented in Fig. 11, if the reference is in place, and in Fig. 12, if the load specimen is in place. In presence of the reference there is no actual shielding material inserted between the two halves of the coaxial line and the electromagnetic field is free to propagate throughout the length of the coaxial sample holder. Once the load is inserted, its shielding ability prevents the propagation of the electromagnetic radiation, as shown in Fig. 12, where an homogeneous element with a thickness of $3 \mathrm{~mm}$ and conductivity of $3161.45 \mathrm{~S} / \mathrm{m}$ is acting as a shield against the electromagnetic waves.

The results of the simulations are given in Fig. 13,14. To obtain accurate results a finer mesh was considered for Specimen A. For this sample a mesh of 32 elements with element's length in z direction of $\simeq 0.03 \mathrm{~mm}$ was used, as a compromise between computational costs and output results. For specimen B a mesh of 29 elements with element's length in z direction of $\simeq 0.10 \mathrm{~mm}$ was used. In average the simulation results are higher in SE compared to the experimental one; however, in the range considered for the simulations, between 0 and $400 \mathrm{~Hz}$, it was possible to obtain curves comparable to those measured experimentally. For specimen A the simulation with a sample of $0.85 \mathrm{~mm}$ in thickness and $12954.21 \mathrm{~S} / \mathrm{m}$ differs in average from the measured values of $\simeq 1.96 \mathrm{~dB}$, while the simulation with a sample of $1 \mathrm{~mm}$ and $10200.65 \mathrm{~S} / \mathrm{m}$ differs in average of $\simeq 1.95 \mathrm{~dB}$. The effect of thickness variation is minimal and slightly affects the slope decreasing it. A smaller thickness affects the absorption loss, and in the simulation with $0.85 \mathrm{~mm}$ the reflection loss compensates for the decrease in absorption. 


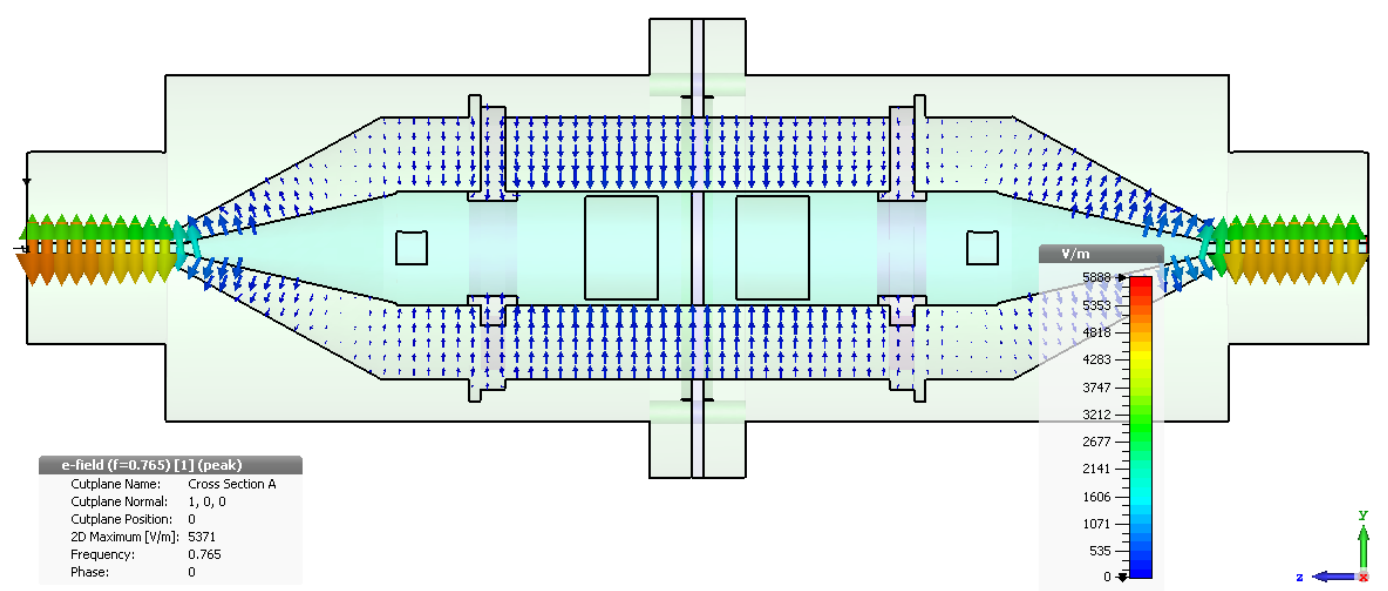

Fig. 11: Electric field propagation in presence of the reference specimen.

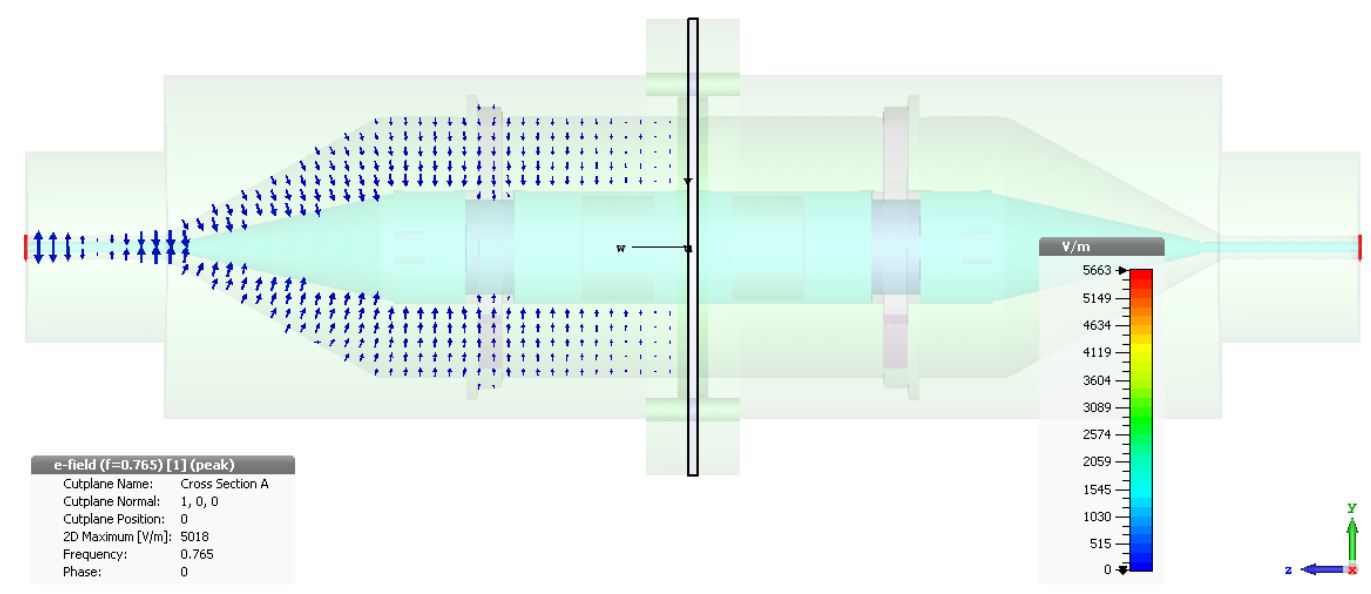

Fig. 12: Electric field propagation in presence of the load specimen.

The more the frequency increases the more the curves obtained seem to converge to the experimental results.

A different trend seems to be applied in the case of specimen B. Also in this case the difference in thickness causes minor variation in the slope of the curve, whereas specimen B has a greater thickness compared to specimen A and therefore the impact of a change in this variable may be less significant. However, the simulations obtained have a greater slope compared to the experimental curve, the linear trendline shows a slope of $\mathrm{m}=0.086$ compared to the linear trendline slope $\mathrm{m}=0.045$ of the experimental curve. The differences between the simulations and the experimental data are probably related to the multiple reflection mechanism that takes place because of impedance mismatches at the various interfaces present inside the composite material which is not considered. The reflection loss parameter decreases with increasing frequency, while the absorption loss increases at high frequencies 


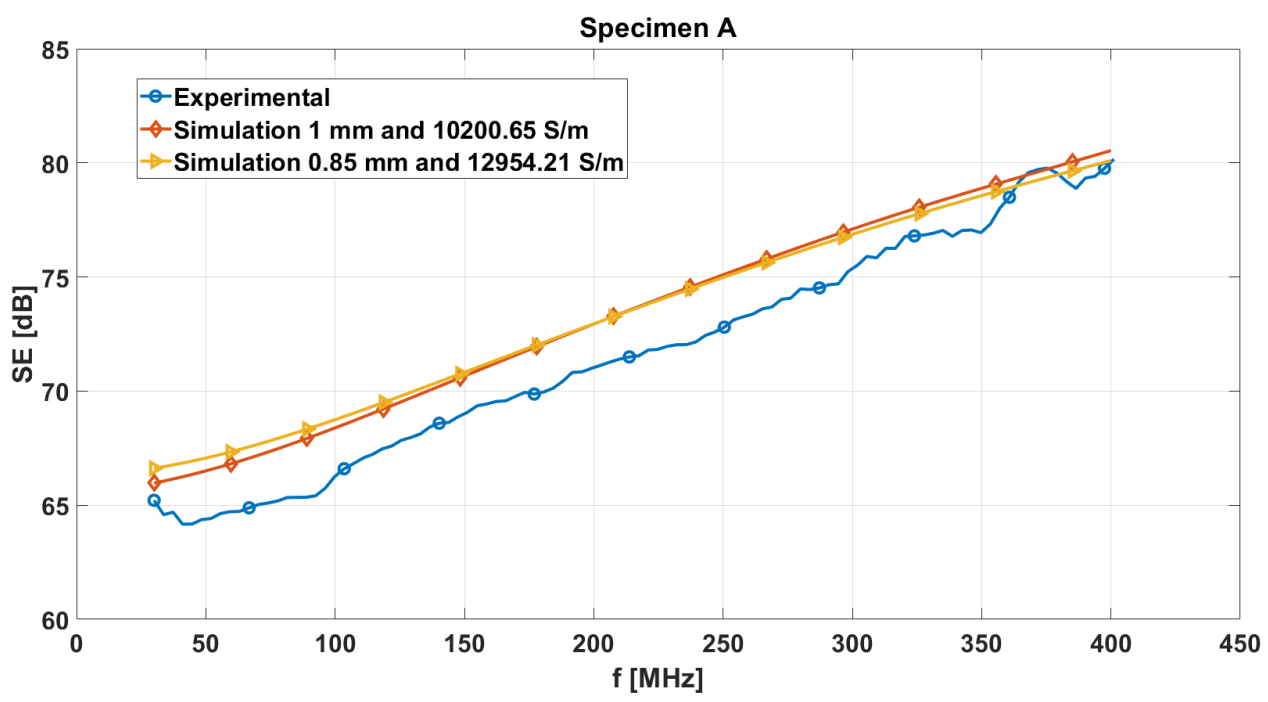

Fig. 13: Shielding effectiveness of Specimen A, experimental vs simulations.

and becomes the primary shielding mechanism. Therefore the more the frequency increases, the more the influence of $S E_{A}$ becomes important. In the curves obtained from the simulation the SE becomes greater than the one measured experimentally increasing the frequency. The $S E_{A}$ may be overestimated because of the lack of a negative correction factor represented by the $S E_{M R}$ parameter. Although the

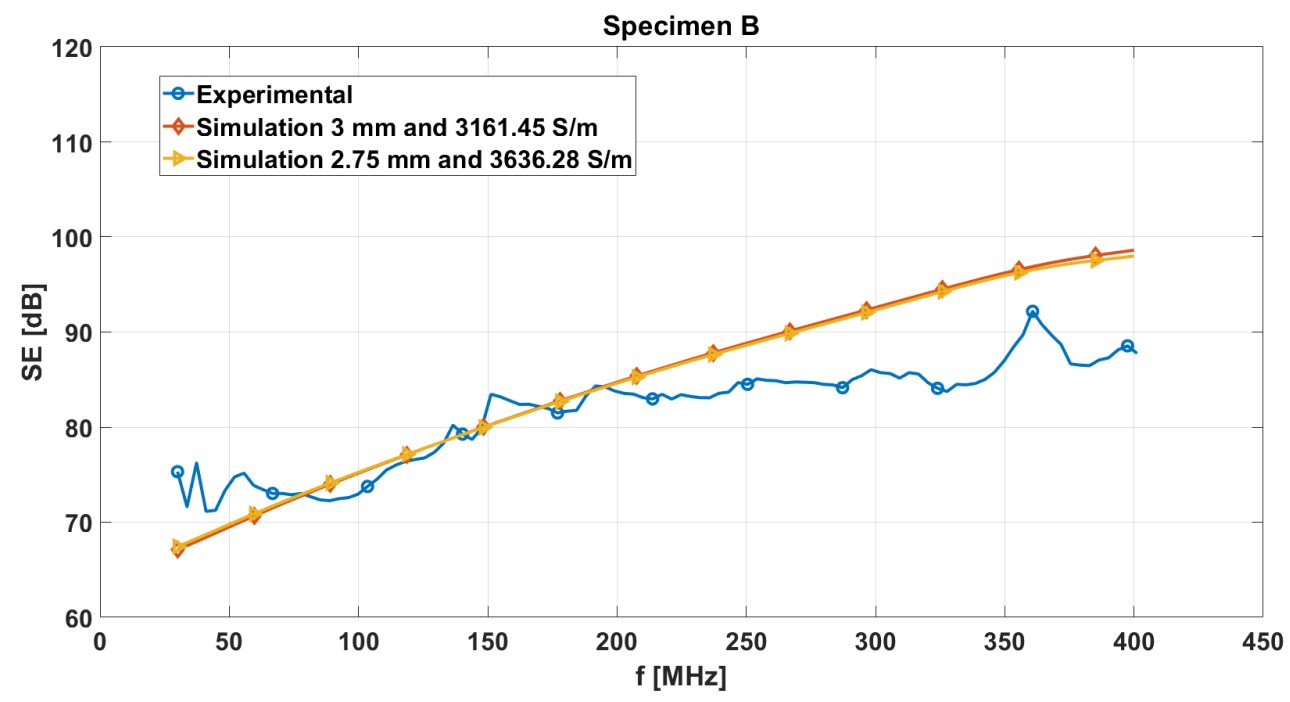

Fig. 14: Shielding effectiveness of Specimen B, experimental vs simulations.

thickness in sample B is greater and a certain absorption loss is expected, the composite material is not homogeneous and contains several boundaries within itself, due to the presence of fibres and resin. Another cause for this behaviour is related to Simon formalism where three-dimensional isotropy of resistivity is assumed; however, both mechanisms (reflection and absorption) are related with in- 
plane and out-of-plane resistivity, which are not always the same. The assumption of homogeneous resistivity works better with smaller thicknesses. Furthermore, it must be taken into consideration that the conductivity was calculated as the average value in the specified range of frequencies.

The overall results of the comparisons between simulations and experimental data suggest that, knowing the conductivity of a thin plate of laminate, the simulations can be used to determine its shielding effectiveness, without resorting to experimental tests. The shielding effectiveness theory of a conductive plate, Eqs. $(9,12,21)$, can be applied within certain limitations to composite materials with filler content over the percolation threshold, i.e. with conductivities of the order of $10^{3}-10^{4} \mathrm{~S} / \mathrm{m}$. These limitations are represented by the number of layers of the laminate. In this study is shown that with a number of layers between 4 and 8 the simulations are comparable with the experimental measurements.

\section{Conclusion}

The information described in this paper is focused on the EMI shielding effectiveness of CFRP composites and on the calculation of the SE parameter of these materials knowing their volume resistivity. The results of the experimental tests show a significant ability to obstruct electromagnetic radiation. A simple layer of $2 / 2$ twill $3 \mathrm{k}$ dry carbon fibres fabric is able to shield more than $99.999 \%$ of the electromagnetic radiation. In Layered carbon fibre/epoxy samples the presence of the resin reduces the contact between fibres, therefore the SE of CFRP composites increases with a higher fibre volume fraction, which ensures a greater continuity of conductive filler along the length and the thickness of the material. A larger thickness and a greater number of fabric layers are not therefore an indication of higher conductivity, as shown in the case of specimen B, which is always related to the fibre volume fraction. However, the specimen with more layers exhibits a greater protection against electromagnetic radiation, proving that the shielding effectiveness is not only related to the conductivity of the material, but is also dependent on the power dissipated along the thickness and on the power reflected at different interfaces.

CST Microwave Studio provides an efficient platform for performing simulations of shielding materials. The simulation work described applies to the less generic case of CFRP materials, which were presented as simple geometrical elements with homogeneous conductivity properties. For $1 \mathrm{~mm}$ thick specimen $\mathrm{A}$, the $3 \mathrm{D}$ model offer a level of accuracy less than $\simeq 2 \mathrm{~dB}$ compared to the experimental

tests results. So it is possible to neglect the effect of a separate modelling for resin and fibres and the presented model can be used as a valid approximation of the reality in this range of frequencies. The model proves also that the re-reflections losses can be disregarded to a certain extent and that the approximation calculated with Simon formalism is adequate to describe the problem. An increase in 
thickness favours the difference between the simulated shielding effectiveness and experimental results. In a thicker specimen made of carbon fibres/epoxy composite, with more layers of fabric, possibly the number of interfaces at different impedance is greater, causing a more significant re-reflections mechanism. In this case it may be appropriate to take into account the separate influence of matrix and fibres at mesoscopic scale. Another factor, that may have affected the output, depends on the application of Simon formalism to calculate the SE as an average of values.

\section{Acknowledgments}

This work was funded by the INNOVATIVE doctoral programme. The INNOVATIVE programme is partially funded by the Marie Curie Initial Training Networks (ITN) action (project number 665468) and partially by the Institute for Aerospace Technology (IAT) at the University of Nottingham.

The work was supported through the provision of a cooperation license for the CST Studio Suite by CST (Dassault Systemes).

\section{References}

[1] Viskadourakis Z, Vasilopoulos K, Economou E, Soukoulis CM, Kenanakis G. Electromagnetic shielding effectiveness of 3d printed polymer composites. Applied Physics A 2017;123(12):736-42.

[2] Geetha S, Satheesh Kumar K, Rao CR, Vijayan M, Trivedi D. Emi shielding: Methods and materials-a review. Journal of applied polymer science 2009;112(4):2073-86.

[3] Leitgeb N, Schröttner J, Böhm M. Does "electromagnetic pollution" cause illness? Wiener Medizinische Wochenschrift 2005;155(9-10):237-41.

[4] Balmori A. Electromagnetic pollution from phone masts. effects on wildlife. Pathophysiology 2009;16(2-3):191-9.

[5] Rea WJ, Pan Y, Fenyves EJ, Sujisawa I, Suyama H, Samadi N, et al. Electromagnetic field sensitivity. Journal of Bioelectricity 1991;10(1-2):241-56.

[6] Kubík Z, Skála J. Shielding effectiveness simulation of small perforated shielding enclosures using fem. Energies 2016;9(3):129-40.

[7] Yang S, Lozano K, Lomeli A, Foltz HD, Jones R. Electromagnetic interference shielding effectiveness of carbon nanofiber/lcp composites. Composites Part A: applied science and manufacturing $2005 ; 36(5): 691-7$.

[8] Ott HW. Electromagnetic compatibility engineering. John Wiley \& Sons; 2011. 
[9] Håkansson E, Amiet A, Kaynak A. Electromagnetic shielding properties of polypyrrole/polyester composites in the 1-18 ghz frequency range. Synthetic metals 2006;156(14-15):917-25.

[10] Thomassin JM, Jerome C, Pardoen T, Bailly C, Huynen I, Detrembleur C. Polymer/carbon based composites as electromagnetic interference (emi) shielding materials. Materials Science and Engineering: R: Reports 2013;74(7):211-32.

[11] Chung D. Materials for electromagnetic interference shielding. Journal of Materials Engineering and performance 2000;9(3):350-4.

[12] Schelkunoff SA. Electromagnetic waves. New York : D. Van Nostrand Company; 1943.

[13] Schulz RB, Plantz V, Brush D. Shielding theory and practice. IEEE Transactions on Electromagnetic Compatibility 1988;30(3):187-201.

[14] Bigg D. The effect of chemical exposure on the emi shielding of conductive plastics. Polymer composites 1987;8(1):1-7.

[15] Colaneri NF, Schacklette L. Emi shielding measurements of conductive polymer blends. IEEE transactions on instrumentation and measurement 1992;41(2):291-7.

[16] Jou W, Wu T, Chiu S, Cheng W. Electromagnetic shielding of nylon-66 composites applied to laser modules. Journal of electronic Materials 2001;30(10):1287-93.

[17] Yang Y, Gupta MC, Dudley KL. Towards cost-efficient emi shielding materials using carbon nanostructure-based nanocomposites. Nanotechnology 2007;18(34):345-8.

[18] Tzeng SS, Chang FY. Emi shielding effectiveness of metal-coated carbon fiber-reinforced abs composites. Materials Science and Engineering: A 2001;302(2):258-67.

[19] Luo X, Chung D. Electromagnetic interference shielding using continuous carbon-fiber carbonmatrix and polymer-matrix composites. Composites Part B: Engineering 1999;30(3):227-31.

[20] Wu J, Chung D. Increasing the electromagnetic interference shielding effectiveness of carbon fiber polymer-matrix composite by using activated carbon fibers. Carbon 2002;40(3):445-7.

[21] Cao MS, Song WL, Hou ZL, Wen B, Yuan J. The effects of temperature and frequency on the dielectric properties, electromagnetic interference shielding and microwave-absorption of short carbon fiber/silica composites. Carbon 2010;48(3):788-96.

[22] Das N, Khastgir D, Chaki T, Chakraborty A. Electromagnetic interference shielding effectiveness of carbon black and carbon fibre filled eva and nr based composites. Composites part A: applied science and manufacturing 2000;31(10):1069-81. 
[23] Al-Saleh MH, Sundararaj U. Electromagnetic interference shielding mechanisms of cnt/polymer composites. Carbon 2009;47(7):1738-46.

[24] Balageas D, Fritzen CP, Güemes A. Structural health monitoring; vol. 90. John Wiley \& Sons; 2010.

[25] Chung D. Electromagnetic interference shielding effectiveness of carbon materials. Carbon 2001;39(2):279-85.

[26] Li L, Chung D. Electrical and mechanical properties of electrically conductive polyethersulfone composites. Composites 1994;25(3):215-24.

[27] Fouladgar J, Wasselynck G, Trichet D. Shielding and reflecting effectiveness of carbon fiber reinforced polymer (cfrp) composites. In: Proceedings of 2013 URSI International Symposium on Electromagnetic Theory (EMTS). IEEE; 2013, p. 104-7.

[28] Jana P, Mallick A, De S. Effects of sample thickness and fiber aspect ratio on emi shielding effectiveness of carbon fiber filled polychloroprene composites in the $\mathrm{x}$-band frequency range. IEEE transactions on electromagnetic compatibility 1992;34(4):478-81.

[29] Jana P, Mallick A, De S. Electromagnetic interference shielding by carbon fibre-filled polychloroprene rubber composites. Composites 1991;22(6):451-5.

[30] Jana P, Mallick A, De S. Electromagnetic interference shielding effectiveness of short carbon fibrefilled polychloroprene vulcanized by barium ferrite. Journal of materials science 1993;28(8):2097104.

[31] Jana P, Mallick A, De S. Electrically conductive rubber and plastic composites with carbon particles or conductive fibres. Short fibre-polymer composites, Cambridge: Woodhead 1996;:16891.

[32] Koledintseva MY, Drewniak JL, DuBroff RE, Rozanov KN, Archambeault B. Modeling of shielding composite materials and structures for microwave frequencies. Progress In Electromagnetics Research 2009;15:197-215.

[33] Koledintseva M, Drewniak J, Zhang Y, Lenn J, Thoms M. Modeling of ferrite-based materials for shielding enclosures. Journal of Magnetism and Magnetic Materials 2009;321(7):730-3.

[34] Wieckowski TW, Janukiewicz JM. Methods for evaluating the shielding effectiveness of textiles. Fibres \& Textiles in Eastern Europe 2006;5(59):18-22. 
[35] Ghodgaonkar DK, Ali NA, Giubbolini L. Microwave nondestructive testing of composite materials using free-space microwave measurement techniques. In: 15th World Conference on NonDestructive Testing. 2000, p. 15-21.

[36] Kovar S, Valouch J, Urbancokova H, Adamek M, Mach V. Simulation of shielding effectiveness of materials using cst studio. WSEAS Transactions on Communications 2017;16:131-6.

[37] Munteanu I, Hänninen I. Recent advances in cst studio suite for antenna simulation. In: 6th European Conference on Antennas and Propagation (EUCAP). IEEE; 2012, p. 1301-5.

[38] Rütschlin M, Wittig T. State of the art antenna simulation with cst studio suite. In: 9th European Conference on Antennas and Propagation (EuCAP). IEEE; 2015, p. 1-5.

[39] Fang CH, Zheng S, Tan H, Xie D, Zhang Q. Shielding effectiveness measurements on enclosures with various apertures by both mode-tuned reverberation chamber and gtem cell methodologies. Progress In Electromagnetics Research 2008;2:103-14.

[40] Hao J, Qi P, Fan J, Guo Y. Analysis of shielding effectiveness of enclosures with apertures and inner windows with tlm. Progress In Electromagnetics Research 2013;32:73-82.

[41] Belkacem FT, Bensetti M, Boutar AG, Moussaoui D, Djennah M, Mazari B. Combined model for shielding effectiveness estimation of a metallic enclosure with apertures. IET Science, Measurement \& Technology 2011;5(3):88-95.

[42] Celozzi S, Araneo R. Alternative definitions for the time-domain shielding effectiveness of enclosures. IEEE Trans Electromagnetic Compatibility 2014;56(2):482-5.

[43] Park KY, Lee SE, Kim CG, Han JH. Application of mwnt-added glass fabric/epoxy composites to electromagnetic wave shielding enclosures. Composite structures 2007;81(3):401-6.

[44] Park KY, Lee SE, Lee WJ, Kim CG, Han JH. The application of fiber-reinforced composites to electromagnetic wave shielding enclosures. Composites Research 2006;19(3):1-6.

[45] Al-Saleh MH, Sundararaj U. Electromagnetic interference shielding mechanisms of cnt/polymer composites. Carbon 2009;47(7):1738-46.

[46] Saadi H, Oussaid R. Materials effect on shielding effectiveness. In: International Conference on Signal Processing and Communications. IEEE; 2007, p. 999-1002.

[47] Liu Z, Bai G, Huang Y, Ma Y, Du F, Li F, et al. Reflection and absorption contributions to the electromagnetic interference shielding of single-walled carbon nanotube/polyurethane composites. Carbon 2007;45(4):821-7. 
[48] Saini P, Choudhary V, Singh B, Mathur R, Dhawan S. Polyaniline-mwcnt nanocomposites for microwave absorption and emi shielding. Materials Chemistry and Physics 2009;113(2-3):919-26.

[49] Quievy N, Bollen P, Thomassin JM, Detrembleur C, Pardoen T, Bailly C, et al. Electromagnetic absorption properties of carbon nanotube nanocomposite foam filling honeycomb waveguide structures. IEEE Transactions on Electromagnetic Compatibility 2012;54(1):43-51.

[50] Simon RM. Emi shielding through conductive plastics. Polymer-Plastics Technology and Engineering 1981;17(1):1-10.

[51] Lu G, Li X, Jiang H. Electrical and shielding properties of abs resin filled with nickel-coated carbon fibers. Composites Science and Technology 1996;56(2):193-200.

[52] Cui W, Du F, Zhao J, Zhang W, Yang Y, Xie X, et al. Improving thermal conductivity while retaining high electrical resistivity of epoxy composites by incorporating silica-coated multi-walled carbon nanotubes. Carbon 2011;49(2):495-500.

[53] Chung D. Carbon materials for structural self-sensing, electromagnetic shielding and thermal interfacing. Carbon 2012;50(9):3342-53.

[54] Wypych G. Handbook of fillers. Elsevier; 2016.

[55] Rütschlin M, Wittig T. State of the art antenna simulation with cst studio suite. In: 9th European Conference on Antennas and Propagation (EuCAP). IEEE; 2015, p. 1-5.

\section{Nomenclature}

E: Electric field

H: Magnetic field

$\eta_{0}$ : Impedance of the free space

$\eta_{w}$ : Impedance of the incident wave

$\eta_{S}$ : Impedance of the shield

$f$ : Frequency

$\omega$ : Angular frequency

$\varepsilon$ : Permittivity

$\mu$ : Magnetic permeability

$\varepsilon_{0}, \mu_{0}$ : Permittivity and magnetic permeability of free space

$\varepsilon_{r}, \mu_{r}$ : Relative permittivity and relative permeability

$\sigma$ : Electrical conductivity 
$\delta$ : Skin depth

$\gamma$ : Propagation constant

$t$ : Thickness of the material

$P$ : Electromagnetic signal/transmission power

$P_{i}$ : Input power

$P_{r}$ : Power reflected

$P_{t}$ : Power transmitted

$P_{a}$ : Power absorbed

$R$ : Reflectance

A: Absorbance

$T$ : Trasmittance

$S_{i j}$ : Scattering parameters

$S E$ : Shielding effectiveness

$S E_{R}$ : Reflection loss

$S E_{A}$ : Absorption loss

$S E_{M R}:$ Re-reflection loss 